\title{
Prévention et protection contre le risque d'inondation des installations sur les sites sensibles de France Telecom
}

\author{
Safety and protection of France Telecom sites exposed to flood risk \\ Par F. Pierre \\ France Telecom, Branche Ressources, D.I.A.
}

France Telecom is subject to public services commitments and has to think of safety and protection against flood risk, which threatens switching and transmission equipements. Diagnosis of risk sites, collecting information, setting up a list of prevention actions, protecting the buildings and technical installations, are some of the measures taken. After Nîmes and Vaison-la-Romaine disasters, the decisions were taken more rapidly.

\section{I INTRODUCTION}

De violentes inondations ont entraîné au cours des dernières années des dommages au patrimoine et aux installations techniques et des perturbations au service rendu à la clientèle ; ce préjudice est double et atteint l'exploitant et les usagers.

Une prévention renforcée est donc nécessaire, d'autant plus que son coût sera presque toujours inférieur à celui représenté par les travaux de remise en état et les pertes de recettes résultant du sinistre.

France Télécom, assujetti à une obligation de service public, est donc contraint de prendre en compte le risque d'inondation dont les effets peuvent avoir des conséquences désastreuses.

Ce bref exposé ne se limitera pas au seul risque en région parisienne, mais englobera l'ensemble de la métropole où l'exploitant est présent en tous points.

\section{II $\square$ LE DIAGNOSTIC : TYPE DE RISQUE À IDENTIFIER}

Il importe, en préalable, de diagnostiquer le plus précisément possible quel type de risque peut nous menacer. Ces risques sont de quatre sortes :

- le débordement de cours d'eau dû essentiellement à de fortes et durables précipitations,

- le ruissellement d'orage, ponctuel et d'autant plus dangereux que la zone exposée est urbanisée,

- le refoulement d'égout, généralement par sous-dimensionnement du réseau de collecte des eaux pluviales,

- la remontée de nappe phréatique, due à l'intensité de la pluviométrie sur une période plus ou moins longue, étalée dans le temps.

En remarque on peut ajouter que, lorsqu'un bâtiment a été inondé, il n'est plus nécessaire de s'interroger sur sa vulnérabilité qui vient d'être démontrée.

En revanche, pour tous les autres sites qui ont pu être épargnés jusqu'à maintenant, la question de savoir s'ils sont ou non vulnérables et dans quelle mesure, doit être posée.

\section{III — LA DÉMARCHE À ENTREPRENDRE}

La démarche à entreprendre consiste à rassembler les connaissances et les éléments physiques afin que l'approche du risque potentiel soit rendue possible et que les mesures pour s'en prémunir puissent être décidées. En voici les étapes principales :

\section{- Etude de la cartographie et des plans:}

Les premières recherches devront procurer la cartographie et les données hydrologiques de la région ainsi que le dossier de plans du bâtiment (DOE). Il faudra également vérifier la présence ou la proximité d'une voie d'eau, la déclivité des lieux, la présence ou non d'obstacles s'opposant au libre écoulement des eaux comme une voie ferrée par exemple.

Les niveaux atteints par les crues sont le plus souvent exprimés en m. NGF. Il faudra donc exiger le rattachement du bâtiment au nivellement général de la France.

Enfin il est indispensable que l'ensemble des caractéristiques propres au bâtiment soient parfaitement connues : niveaux, nature des sous-sols, radier, cuvelage, réseaux souterrains, drains et pompes de relevage...

- Entrevue avec le représentant PER de la DDE : 
Ce contact doit être établi préalablement à toute construction sur un terrain situé dans une zone réputée inondable. Cet agent sera en mesure d'apporter des renseignements précis et fiables sur les risques d'inondation de la zone concernée.

\section{- Visite minutieuse du site :}

Cet aspect de la démarche s'inscrit volontairement dans une approche préventive.

En effet, si un bâtiment a déjà été inondé (quelle qu'en soit la cause), les décisions de sauvegarde et de protection seront d'autant plus "faciles" à prendre.

En revanche, lorsqu'un bâtiment n'a jamais subi d'inondation, mais que l'on craint un risque potentiel, il faudra déterminer le mieux possible l'importance du risque et les points singuliers du bâtiment qui représentent des faiblesses par rapport aux venues d'eau.

\section{- Principales mesures à prendre :}

Elles concernent, en priorité, les centres téléphoniques importants et les centres principaux d'exploitation. Ces mesures et les travaux qui en résultent seront listés par ordre d'importance pour répondre à la permanence du service public.

Ce sont les installations téléphoniques elles-mêmes qui seront protégées (la commutation et la transmission) ainsi que les équipements techniques qui contribuent à leur bon fonctionnement. Ces équipements sont qualifiés de "environnement technique des systèmes de télécommunications". Ils regroupent l'alimentation en énergie, le secours en énergie, la climatisation et le traitement de l'air ambiant, la détection et la protection-incendie.

\section{- Décision de la Direction du réseau:}

La décision de la direction du réseau sera dictée par la nature et la répartition des sites stratégiques et dont on peut penser - a priori - qu'ils n'ont pas été construits en zone inondable.

Si tel était le cas a contrario, la décision à prendre relèverait d'un choix : soit on maintient le bâtiment et ses installations sur le site en mettant en œuvre les mesures de protection nécessaires, soit on transfère le bâtiment et ses installations sur un nouveau site, non vulnérable, en assurant le coût correspondant.

\section{IV — LES MESURES DE PRÉVENTION}

Le résumé que l'on peut faire de ces mesures tient à :

- la connaissance préalable des PERI (plan d'exposition aux risques d'inondation),

- la connaissance préalable des PSS (plan des surfaces submersibles), des communes sur lesquelles se trouvent des bâtiments du réseau de télécommunications,

- la connaissance du périmètre des risques, en référence à l'article R III - 3 du décret 77-755 du 07/07/1977,

- la référence aux circulaires récentes qui sont celles :

$$
\begin{aligned}
& \text { - du 24/01/1994 } \\
& \text { - du 19/07/1994 } \\
& \text { - et n } 94-69 \text { du 16/08/1994, }
\end{aligned}
$$

- la décision de proscrire toute construction nouvelle et même toute extension de bâtiment existant en zone inondable.

\section{I LES MESURES DE PROTECTION ET DE SÉCURISATION}

Parmi les plus importantes, on peut citer :

- l'obturation étanche des pénétrations : les ouvertures diverses, telles que les passages de câbles et de tuyauteries, situées au-dessous du niveau de la crue centennale devront être soigneusement obturées,

- le niveau des prises d'air au-dessus de celui de la crue centennale ; ces baies de ventilation ne doivent pas servir d'exutoire à une crue éventuelle,

- la pose de clapets anti-retour sur les branchements à l'égoût public, complétée chaque fois que possible par une vanne de fermeture,

- la surveillance de l'état des cuvelages et la mise en place de pompes de relevage (seules ou en réseau) munies de détecteurs de venue d'eau et d'un système d'alarmes,

- le relèvement des matériels sensibles sur des niveaux du bâtiment hors d'atteinte de la crue éventuelle. De telles opérations ont déjà été réalisées à France Télécom soit en positionnant ces équipements sur des socles construits dans les locaux vulnérables, soit en déplaçant ces équipements du sous-sol au rez-de-chaussée par exemple.

\section{VI — L'ÉTAT DES LIEUX}

L'acuité de la prise de conscience du risque d'inondation a été considérablement renforcée par la catastrophe de Nîmes en octobre 1988 où France Télécom a été durement atteint et où on a déploré une importante interruption du trafic, touchant plusieurs milliers de lignes.

Quelques années plus tard, la catastrophe de Vaison-laRomaine a de nouveau rappelé que des zones récemment urbanisées pouvaient être le théâtre d'inondations épouvantables allant jusqu'à mettre en péril les vies humaines.

Les analyses de la situation conduites dans les rapports PONTON et BOURGES ont constitué le support permettant aux responsables de la sécurité à France Télécom d'engager la réflexion et de décider des actions à entreprendre sur ce sujet d'enjeu primordial.

Le travail entrepris sur les zones réputées vulnérables du sud de la France a été étendu à l'ensemble du territoire national et les opérations de prévention comme de protection et de sauvegarde ont fait l'objet de chiffrages et d'estimations d'enveloppes budgétaires.

Les responsables régionaux ont mis en œuvre ou poursuivent la mise en cuvre du plan de mise à niveau des sites de production considérés comme relevant du risque d'inondation. 